\title{
Overpricing and Hidden Costs of Structured Bonds for Retail Investors: Evidence from the Danish Market for Principal Protected Notes
}

\author{
Peter Løchte Jørgensen and Henrik Nørholm and David Skovmand* \\ Finance Research Group \\ Department of Economics and Business \\ Business and Social Sciences \\ Aarhus University \\ Fuglesangs Allé 4 \\ DK-8210 Aarhus V \\ DENMARK \\ E-mail: plj@asb.dk / henrn@asb.dk / davids@asb.dk
}

Current version: June 13, 2011

JEL Classification Codes: G12 (Asset pricing), G13 (Contingent pricing).

Keywords: Structured products, fair valuation, pricing efficiency, exotic option pricing, cost analysis.

\footnotetext{
${ }^{*}$ The authors gratefully acknowledge useful suggestions and comments from Ken L. Bechmann, Bent Jesper Christensen, Bjarne Astrup Jensen, Mikkel Svenstrup, and from participants at the 2009 EFMA Doctoral Seminar in Milan, the 2010 Nordic Finance Network Research Workshop in Lund, and a seminar at HEC Montréal's Finance Department in February 2011. The paper's empirical work builds on a significantly expanded version of a database which was initiated by Anne-Sofie Reng Rasmussen.
} 


\title{
Overpricing and Hidden Costs of Structured Bonds for Retail Investors: Evidence from the Danish Market for Principal Protected Notes
}

\begin{abstract}
This paper studies the cost structure and pricing efficiency of principal protected notes (PPNs) from the Danish retail market. Our data set consists of detailed information on almost 400 Danish issues of PPNs during the period from 1998 to 2009. Comparing actual offer prices with theoretical fair values we find that on average PPNs are overpriced by about $6 \%$. Only half of the overpricing can be explained by the costs disclosed by sellers at the time of issuance.

At the individual instrument level we find time to maturity and indicators of product complexity to be important determinants of costs and of the degree of overpricing, but other factors such as arranger and issuer size play a part as well. The degree of overpricing of PPNs has declined over time, but the unexplained cost component - hidden costs has not.
\end{abstract}

\section{Introduction}

This paper provides new evidence on the pricing of structured products for retail investors. The market for such products has grown rapidly during the past 10-15 years, and although the recent financial crisis put this growth to a pause, the popularity of structured products is currently picking up again, and the size of the market is significant in many countries throughout the world. According to recent estimates gross sales of structured retail investment products amounted to EUR 174.2bn in Europe, USD 179.8bn in the Asia-Pacific market, and to USD $65.1 \mathrm{bn}$ in North America during the year 2010 . $^{1}$

The growing popularity and economic significance of the retail market for structured products have naturally attracted the attention of academics, the financial media, and sometimes also of regulators and supervisory authorities. Much of this attention has been negative. Structured products have typically been criticized for being excessively complex as well as for being too costly and overpriced, and regulators have in some cases responded accordingly. In Norway, for example, following a heated debate about a string of scandalized structured

\footnotetext{
${ }^{1}$ These numbers were obtained from the "Analysis \& Reports" database at www.structuredretailproducts.com.
} 
investment products, the government in March 2008 practically banned the sale of complicated financial products to retail investors. ${ }^{2}$ In the US there have long been severe restrictions on investment banks' sale of structured products to unsophisticated investors, see e.g. Bethel and Ferrel (2006).

As a natural consequence of the concerns raised in relation to structured products a number of papers from the academic literature have studied the pricing of structured retail products in various markets. For example, products from the US market have been analyzed in Chen and Kensinger (1990), Chen and Sears (1990), Benet, Giannetti, and Pissaris (2006), Chen and $\mathrm{Wu}$ (2007), and in Henderson and Pearson (2007). The Swiss and German markets for structured retail products are also large and active, and this is reflected in the amount of research using data from these markets, e.g. Wasserfallen and Schenk (1996), Burth, Kraus, and Wohlwend (2001), Wilkens, Erner, and Roder (2003), Stoimenov and Wilkens (2005), Grunbichler and Wohlwend (2005), Wallmeier and Diethelm (2009), Rathgeber and Wang (2010), Ruf (2010), and Baule and Tallau (2011). Products from the Dutch market are analyzed in Szymanowska, Horst, and Veld (2009), and Bennett, Chen, and McGuinness (1996) is an early study of some products issued in Hong Kong. With just one exception - Wasserfallen and Schenk (1996) - all of the papers cited above find the type of structured retail products that they analyze to be overpriced at the time of issuance.

The purpose of the present paper is to contribute further to this string of literature by presenting results and empirical evidence in relation to the voluminous Danish market for a highly homogeneous class of structured retail products known as Principal Protected Notes. Using a unique hand-collected data set consisting of all relevant information regarding the almost full population sample, i.e. nearly 400 issues, of principal protected notes issued during the past 12 years, and by applying widely recognized theoretical pricing techniques to as many of the products in the data set as possible, we find strong evidence of overpricing of principal protected notes in the Danish market. More precisely, we find an average overpricing of about $6 \%$ and while this is in fine accordance with the order of magnitude of the mispricing reported in other studies, our empirical results emerge on the basis of one of the largest data sets that has so far been applied in analyses of structured products for retail investors. In addition, our database contains a large fraction of currency/FX based products whereas previous research has concentrated almost exclusively on equity-based products.

\footnotetext{
${ }^{2}$ See e.g. press release $4 / 2008$ at www.finanstilsynet.no.
} 
Compared to previous studies we also take the analysis a step further by decomposing total costs into costs that are disclosed by the seller at issuance and an unexplained remainder which we denote hidden costs. Much to our surprise we find that only half of all costs are disclosed to investors, or in other words, that true total costs are more than double of what investors are told at the time of investment. This somewhat worrying finding is new to the literature, and it is quite robust to model error and to mis-estimation of pricing model parameters.

Another important contribution of our paper is the finding that to a large extent costs of individual products can be explained by product specific characteristics. Via multivariate regression analysis we find factors relating to products' time to maturity, arranger size, and complexity to be main determinants of product costs and the degree of overpricing.

The remainder of the paper is organized as follows. In the next section we provide additional background and information on the structure of the securities that we study in this paper. Section 3 presents and briefly discusses our database. Our research methods are explained in Section 4, and Section 5 presents the empirical findings. Section 6 concludes.

\section{Background on the securities and the market}

The securities studied in this paper are composite financial products that are structured specifically for sale to retail investors. As we explain in more detail in the data section below, we have collected information and data on almost 400 issues of these remarkably similar structured products from the Danish market in the 12-year period from the beginning of 1998 and until the end of 2009. In addition to being targeted at retail investors the common characteristic of the financial products investigated here is that they are in effect decomposable into two basic elements. The first of these elements is a simple bond. In some cases the bond promises a fixed (and then quite low) annual coupon, but in most cases the bond is a straight zero-coupon bond. The presence of the (zero-coupon) bond in the two-component investment "package" implies a capital guarantee which is effective at maturity. For this reason - and because the bonds are usually of medium term - the products in question are often referred to as Principal Protected Notes or PPNs. ${ }^{3}$ It is without a doubt that the capital guarantee plays

\footnotetext{
${ }^{3}$ Most products (more than $90 \%$ ) in our data set have full protection of principal, i.e. redemption at at least par is guaranteed. The remaining bonds in the sample have either only partial protection of principal or a
} 
an important role in the active marketing of the products.

The second element of the structured bond package is a European-style option which is written on some kind of "index" and which expires at the same time as the bond element. The role of the option element is to provide an upside potential for the investor so that when the two elements are combined, the structured product protects the capital of the investor in poor scenarios where the index falls (and the option element expires out-of-the-money), and it returns the principal plus an upside payoff in good scenarios in which the index appreciates. For this reason a better and more accurate denomination of these bonds would be Principal Protected Index Linked Notes, but in accordance with market terminology we retain the shorter "PPN" to mean exactly that. As we shall document later, the payoff profile of these structured products has appealed increasingly to retail investors in recent years. One can perhaps explain such an implicit demand for portfolio insurance $e^{4}$ by retail investors by the use of loss-aversion based portfolio choice models (see e.g. Tversky and Kahneman (1992) and Bernard and Ghossoub (2010)), but such an analysis is outside the scope of the present paper. $^{5}$

Whereas the bond element is more or less identical for all securities in our sample (coupon rates and time to maturity vary slightly), a lot more creativity is observed in the design of the embedded option where both option type and underlying index vary across the many different issues. To formalize a bit, the above-described common structure of PPNs implies a time $T$ payoff function, $P P N(T)$, of the following general form,

$$
P P N(T)=P+P \cdot \delta \cdot C(T)
$$

where $P$ refers to the guaranteed principal and where $\delta$ is the participation rate of the PPN. Finally, $C(T)$ is the time $T$ payoff of the embedded option. In addition to the maturity payoff of the form described in equation (1) there may, as previously mentioned, be fixed coupon payments $\left\{c_{t_{n}}\right\}$ at times $t_{1}, \ldots, t_{N}=T$.

The option payoff, $C(T)$, can take many different forms, but in most cases it is one that benefits from an increase in an underlying index during the life of the PPN. A particularly protection level which is above par.

${ }^{4}$ Note that by the put-call parity a portfolio consisting of a zero-coupon bond plus a call option on some underlying asset is equivalent to a portfolio of the underlying asset plus an otherwise identical put option on the underlying asset. The latter type of portfolio is often referred to as a portfolio insurance.

${ }^{5}$ Other interesting studies in this area - the optimal design of and demand for structured retail products are Hens and Rieger (2008) and Branger and Breuer (2008). 
simple example would be a plain vanilla call option on the total return of an index such that the full PPN payoff at maturity would be

$$
P P N(T)=P+P \cdot \delta \cdot \max \left(\frac{I_{T}-I_{0}}{I_{0}}, 0\right)
$$

where $I_{0}$ and $I_{T}$ denote the initial and maturity values of the index, respectively.

Other commonly used option types are (total return) spread options - i.e. the option to exchange the return on one asset, $I^{1}$, for the return on another asset, $I^{2}-$ where the PPN maturity payoff is

$$
P P N(T)=P+P \cdot \delta \cdot \max \left(\frac{I_{T}^{1}}{I_{0}^{1}}-\frac{I_{T}^{2}}{I_{0}^{2}}, 0\right),
$$

and Asian options which imply

$$
P P N(T)=P+P \cdot \delta \cdot \max \left(\frac{\frac{1}{M} \sum_{i=1}^{M} I_{t_{i}}-I_{0}}{I_{0}}, 0\right),
$$

where the underlying index is sampled at times $\left\{t_{i}\right\}_{i=1 \ldots M}$. These are merely examples. Our data set also contains many basket structures - often in some form of combination with the above-described structures - as well as even more exotic types of European-style options such as Himalayan options and options with Lookback features. ${ }^{6}$ Often the payoff of the options is also capped. The characteristics of the various exotic options will of course be properly taken into account in our detailed analysis of the products' pricing later in the paper.

A few remarks on the participation rate, $\delta$, are in order at this point. As is apparent from expressions (2)-(4), the participation rate is a constant multiplier that can be said to represent the percentage by which the investor participates in the option payoff. In practice participation rates vary between around 30 percent and several hundred percent, and while a participation rate of 500 percent, say, may sound appealing, it is important to keep in mind that the participation rate is inversely related to the unit value of the embedded option. To understand this, we note that PPNs are almost always issued at a fixed price which is typically a few points above par. Subtracting the "production cost" of the bond element from the fixed issue price leaves a fixed amount for buying options of the desired kind. Since this amount is unlikely to precisely match the price of one option, a scaling factor - the participation rate - must be applied. So when the price of the desired option is low, the participation rate can

\footnotetext{
${ }^{6}$ The reader interested in more detail on the many different exotic option types that can be built into structured products is referred to Kat (2001) and Das (2006).

${ }^{7}$ The two extremes in our data set are $27 \%$ and $1285 \%$.
} 
be high, and vice versa. It follows that it is meaningless to base comparisons of structured bonds solely on their participation rates.

We next briefly describe the life-cycle of a principal protected note. The process of issuing a structured bond involves a number of different agents who, of course, all have an economic incentive to participate in the issue. The central figure in the process is the "arranger" who supposedly possesses the specialized knowledge and expertise necessary for organizing the issue of this type of security. The creativity with respect to the specific design of an issue also normally originates from the arranger. The arranger performs his services on behalf of the formal "issuer" of the PPN. We note (and later document) that issuers of structured bonds are usually financial institutions with a fairly high credit rating. While the issuer is typically an economic entity in need of funding, the issuer may not be willing to assume the risk associated with shorting the option part of the structured bond. Hence, a contract is made with an investment bank to hedge the option element of the product. The investment bank can be paid for this service either up front upon issuance or via a periodic spread. The choice of investment bank is sometimes - but not always - the result of a tendering process where several investment banks are invited to bid on the option based on a so-called term sheet, i.e. a generic description of the desired option. When the arranger, issuer, and investment bank have settled all terms and a prospectus as well as informational and marketing material have been produced, the bond can be put up for sale and a subscription period can begin. During this period, which is typically of 2-4 weeks' duration, the product is actively marketed via the consortium's distribution network (read: local bank branches). At the end of the subscription period the size of the issue can be determined, the participation rate can be set, and the structured bond is listed for trading at the exchange. However, subsequent trades in structured bonds are quite rare events. Bid-ask spreads, if available at all, are very high and PPNs should therefore be considered highly illiquid securities. Finally, at the bonds' maturity date, the investors receive their payoff and the bonds expire and are de-listed.

\footnotetext{
${ }^{8}$ It should be noted here that the participation rate is fixed after the subscription period ends. Thus, investors must base their investment decision on incomplete knowledge of the exact payoff function of the PPN. However, a minimum participation rate is typically specified in the prospectus. If this participation rate cannot be obtained, the issue is normally cancelled.
} 


\section{The data}

This section describes our data set in more detail. We have identified a total of 380 issues of unique PPNs during the period from the beginning of 1998, when the first Danish PPN in our sample was issued, and until the end of 2009. We are confident that only a very limited number of issues may have passed undetected through our search, and our sample of Danish PPNs must therefore be considered as close to the population sample as practically possible. For each of these 380 issues we have collected relevant documents such as the (mandatory) prospectus, fact and information sheets, listing announcements, expiration and redemption announcements, as well as sales brochures and marketing material. This material was obtained from issuers' and arrangers' websites, and from NASDAQ OMX databases. On the basis of the collected material we have created a database containing all information - qualitative as well as quantitative - relevant for answering the research questions at hand. For example, for each issue in our sample the database contains the information listed in Table 1 below.

\section{Table 1:}

\begin{tabular}{|c|c|}
\hline \multicolumn{2}{|c|}{ Key Characteristics of Principal Protected Notes } \\
\hline 1. Name of issue & 10. Participation rate \\
\hline 2. ISIN Code & 11. Protection level \\
\hline 3. Name of arranger & 12. Issue size \\
\hline 4. Name of issuer & 13. Expiry date \\
\hline 5. Issuer rating & 14. Redemption price (if note has expired) \\
\hline 6. Issue date & 15. Currency denomination of note \\
\hline 7. Issue price & 16. Index details \\
\hline 8. Disclosed costs & 17. Option details \\
\hline 9. Coupon rate & \\
\hline \multicolumn{2}{|c|}{$\begin{array}{l}\text { Notes to the table: The ISIN Code is the unique } 12 \text {-character security identifier } \\
\text { Issuer ratings are obtained from Bloomberg and is Moody's rating of the issuer } \\
\text { - not the particular PPN - at the issue date of the PPN. }\end{array}$} \\
\hline
\end{tabular}

Based on this database we have compiled a couple of tables which we present below to pro-

\footnotetext{
${ }^{9}$ See www.nasdaqomxnordic.com.
} 
vide a first descriptive overview of the data set before moving on to more detailed analyses. The first of these tables, Table 2, gives an impression of how the Danish market for PPNs has developed since its inception in 1998. The table documents the strong growth of this market - both in terms of number of issues and nominal issue amounts - up until and including 2006 after which the market cooled off somewhat, perhaps due in part to the financial crisis which began to unfold during 2007. From this table it may be noted that when the market peaked in 2006, a total of 70 different PPNs were issued for a nominal amount exceeding DKK 28bn in that year alone. The total nominal amount of PPNs issued in the data period exceeds DKK $100 \mathrm{bn}^{10}$

Table 2: Development of PPN issues in Denmark, 1998-2009

\begin{tabular}{lrrrrrrrrrrrrr}
\hline Year & 1998 & 1999 & 2000 & 2001 & 2002 & 2003 & 2004 & 2005 & 2006 & 2007 & 2008 & 2009 \\
\hline No. of issues & 3 & 1 & 6 & 15 & 19 & 40 & 32 & 44 & 70 & 61 & 49 & 40 \\
\hline \multicolumn{2}{l}{ Nominal issue size (million DKK) } & & & & & & & & & & \\
\hline Min & 50 & 127 & 62 & 50 & 25 & 16 & 20 & 8 & 26 & 18 & 23 & 4 \\
Max & 75 & 127 & 177 & 210 & 927 & 792 & 681 & 2,416 & 3,044 & 1,570 & 1,148 & 1,110 \\
Average & 63 & 127 & 135 & 119 & 176 & 208 & 220 & 442 & 402 & 296 & 237 & 201 \\
Total & 189 & 127 & 810 & 1,660 & 3,351 & 8,304 & 7,028 & 19,450 & 28,166 & 18,066 & 11,630 & 8,049 \\
\hline
\end{tabular}

Table 3 provides further descriptive statistics across all years for some of the numerical key characteristics of the PPNs in our sample. Quite a few interesting observations regarding the Danish PPN market can be made already from this table. For example, it is seen that the average nominal issue size (DKK $281 \mathrm{mn}$ ) is significantly larger than the median issue size (DKK $152 \mathrm{mn}$ ). This can be explained by the presence of a few very large issues in the data set. ${ }^{11}$ The table also confirms some of our earlier claims: It is seen that the typical time to maturity of a PPN is quite short (3-4 years), that the issue price is typically a few points above par, ${ }^{12}$ that disclosed annual cost percentages - the so-called Annual Percentage Rate of Charge (APR) - are normally in the order of $1 \%$, and that in most cases coupon rates are zero. Looking at the participation rates, we see that these are indeed subject to large variation but with a median equal to $100 \%$. The descriptive statistics for the protection level emphasize

\footnotetext{
${ }^{10}$ EUR $100.00 \approx$ DKK 745.80 .

${ }^{11}$ For an in-depth analysis of the record-breaking 2005 issue (nominal issue size DKK 2.4bn, cf. Table 2) see Skovmand and Jørgensen (2007).

${ }^{12}$ For 312 out of the 380 products in our sample the issue price was in the interval [100; 105$]$.
} 
that most PPNs have full nominal capital protection. Finally it is interesting to note that the median redemption price is also equal to $100 \%$. The implication of this is of course that for a large number of expired PPNs the embedded option has disappointed investors and expired worthless.

Table 3: Descriptive statistics for key characteristics of Danish PPNs

\begin{tabular}{lrrrrr}
\hline & No. obs & Min & Average & Median & Max \\
\hline Nom. issue (m DKK) & 380 & 3.97 & 281.26 & 151.75 & $3,043.50$ \\
Time to maturity (years) & 380 & 0.50 & 3.80 & 3.40 & 11.01 \\
Issue price & 378 & 75.00 & 104.26 & 102.63 & 440.60 \\
Disclosed annual cost \%, APR & 299 & 0.14 & 1.03 & 1.00 & 2.48 \\
Coupon rate (\%) & 341 & 0.00 & 0.70 & 0.00 & 95.00 \\
Participation rate & 248 & 27.00 & 146.07 & 100.00 & $1,285.00$ \\
Protection level & 380 & 55.00 & 99.22 & 100.00 & 120.00 \\
Redemption price & 206 & 80.00 & 111.90 & 100.00 & 222.71 \\
\hline
\end{tabular}

Notes to the table: Averages are equally weighted. 39 issues with some kind of variable coupon rate are excluded from the calculations of statistics regarding the coupon rate. 298 products are entirely without coupon whereas 43 products have a fixed and strictly positive coupon rate. The median of the strictly positive coupon rates is $2 \%$. Note that redemption rates are only available for expired products.

In relation to the non-numerical characteristics it can be noted that 25 different arrangers are represented in the data set. The two largest arrangers represent about half of the PPN market measured by nominal bond values issued. Based on the same measure four arrangers can be characterized as being of medium size. Together these four arrangers hold about a third of the market, whereas the remaining market share is divided between 19 smaller arrangers. A similar categorization can be made of the 30 different issuers that are represented in the data set. Two large issuers have issued a little less than half of all PPNs in our sample, three issuers represent about $25 \%$ of total nominal issues, whereas the remaining $25 \%$ is divided between 25 smaller issuers. With respect to ratings we have already noted that in general issuers are fairly highly rated. To be more specific, we have registered "investment grade" rating of the issuer in all cases where a rating was available (368 out of 380 cases). In 260 
of these cases the rating was the highest possible, i.e. "Aaa".

Finally, and with respect to the bonds' currency denomination, we note that 349 issues are denominated in Danish Kroner (DKK), 24 are in Euros (EUR), 4 are in Norwegian Kroner (NOK), and 3 are in Swedish Kroner (SEK).

\section{Methodology}

As mentioned in the introduction of this article, PPNs have received a fair amount of attention and criticism from many sides including the financial press, the academic community, and consumer/investor organizations. In Denmark the National Bank of Denmark published a critical analysis of this market in its 2nd Quarterly Review in 2007 (see Rasmussen (2007)). The critics of the PPN market have typically focused on the opacity and complexity of the products or they have pointed to the often weak performance of the bonds and thus implicitly questioned the fair pricing of the PPNs at issuance.

While it is difficult to judge objectively whether PPNs are too complex financial products, the question of whether PPNs in the Danish market have been fairly priced at issuance is one that should be possible to answer, and that question is indeed the main focus of the remainder of this article. The current section introduces and discusses central concepts and ideas in relation to our research methodology.

\subsection{Cost measures: Definitions and estimation method}

In order to test the fair pricing of PPNs in the Danish market, we must compare the price at which they are sold with an estimated initial fair value of the products for as many issues as possible. This comparative analysis will be performed at the issue date of the PPNs. Recall that due to the "campaign-sale" conditions under which the products are sold, the issue price of PPNs is in most cases fixed and known well in advance of the actual issue date. ${ }^{13}$ However, in a minority of cases the final issue price is not fixed until shortly before actual issuance. This is the case, for example, for products designed with a fixed participation rate of $100 \%$ and where the issue price is then used for final adjustments, cf. also footnote 8 . In any case we have an observed issue price for each of the 380 PPNs in our database. Since PPNs

\footnotetext{
${ }^{13}$ While the issue price is thus fixed, supply is in practice perfectly elastic.
} 
rarely trade after their inception we cannot effectively test for mispricing of PPNs after their issuance.

While the issue price of PPNs can thus be observed, their fair values must be estimated. In accordance with accounting theory's definition of the fair value concept we will define the fair value of a PPN as "the amount for which it can be exchanged between knowledgeable, willing parties in an arm's length transaction" (see e.g. Scott (2003) and IASB (2004)). When, as in the present case of PPNs, such a value is neither directly observable from an active market nor easily extrapolated from quoted prices of similar assets, then the fair value must be estimated using "valuation techniques consistent with those used by marketplace participants for pricing similar assets or liabilities" (see e.g. FASB (2004)). We will apply the latter principle in the present paper. More precisely, for each PPN in our database we will determine the fair asset value at the issue date, $\widehat{P P N} N_{0}^{\text {Fair }}$, by estimating in turn the initial fair value of the two constituents, i.e. the bond and option element, using well-known and widely accepted valuation techniques based on the ideas of replication and the absence of arbitrage (see e.g. Björk (2009)). The estimated initial fair values of these components will be denoted as $\widehat{B}_{0}^{\text {Fair }}$ and $\widehat{C}_{0}^{\text {Fair }}$, respectively, so that clearly

$$
\widehat{P P N}_{0}^{\text {Fair }}=\widehat{B}_{0}^{\text {Fair }}+\widehat{C}_{0}^{\text {Fair }}
$$

Now, since the promised fixed payments to the bond element are easily identifiable and since issuers have very limited credit risk, the initial fair value of the bond element can be found by calculating the sum of the present value of the perfectly certain promised payments, i.e.

$$
\widehat{B}_{0}^{\text {Fair }}=\sum_{n=1}^{N} c_{t_{n}} \cdot e^{-r_{t_{n}} \cdot t_{n}}+P \cdot e^{-r_{T} \cdot T},
$$

where $c_{t_{n}}$ is the coupon payment at time $t_{n}$ (if any), $P$ is the guaranteed principal, and the $r_{t_{n}}$ 's are the (continuously compounded) risk free zero-coupon interest rates prevailing at the issue date and relating to time $t_{n}$. On the right-hand side of the expression in (6), only the zero-coupon interest rates are not directly observable so these must be estimated from market data. We have used Bloomberg's "Danish LIBOR Zero curve" on the relevant valuation dates for our calculations. This curve consists of continuously compounded zero-coupon rates stripped from LIBOR rates in the short end of the maturity spectrum and from swap rates for longer maturities. 
While estimation of the fair value of the bond elements is thus fairly straightforward, the estimation of the fair value of the option elements is a much harder and more time-consuming task. This is mainly because options are contingent claims with more complex and uncertain payoffs for which models are needed to determine their present value. As already mentioned, there are also many diverse and exotic option types represented in our sample and each of these must be treated individually. Finally, a varying number of model parameters must be specified, estimated and/or calibrated for each single option pricing problem.

In order to be able to overcome the task of pricing as many of the PPNs' embedded options as possible, we choose to work within a classical Black and Scholes (1973) setting where underlying indices follow lognormal diffusions (Geometric Brownian Motions or GBMs) with constant parameters and constant interest rate(s). Interest rates are chosen to match the maturities of the options. We extend the classical Black-Scholes framework to account for multiple and correlated underlying assets, continuous dividends, and quanto adjustments where necessary, but we do not consider more sophisticated option pricing models that include e.g. jumps and/or stochastic volatility and interest rates. This is of course a limitation of the analysis. $^{14}$

The Black-Scholes framework admits closed form solutions for the simpler options such as plain vanilla call options and spread options (Margrabe (1978)), and good approximation formulas have been derived for various types of basket and Asian options (see again Hull (2009)). As explained in Section 2, these option types occur quite frequently in our data set. However, on closer inspection of the option terms one often finds added features such as caps and shorter or longer Asian tails which make the options deviate from the straight plain vanilla options that are priced by closed formulas. In practice we have therefore priced all option elements by Monte Carlo simulation (Boyle (1977)).

As in all practical applications of option pricing models a number of input parameters are needed. Some of these may be read directly from the PPN prospectus (e.g. strike price and time to maturity), some can be obtained from market quotes (e.g. current value of underlying index and interest rates), and some must be estimated. Parameters which must be estimated are primarily volatilities and correlations of underlying indices and currency exchange rates. As regards volatilities, we have collected and used implied volatilities whenever possible since

\footnotetext{
${ }^{14}$ Many option elements in our sample are in fact quantos since underlying indices are e.g. foreign stock indices while the payoff is specified directly in Danish Kroner (DKK). We refer to Hull (2009) for the theory behind quanto adjustments.
} 
these are forward-looking and therefore normally preferred over the alternative of (backwardlooking) historical volatilities. Implied volatilities were obtained from Bloomberg and chosen to reflect the characteristics of the structured products' embedded option as closely as possible. In practice this means that all implied volatilities are market quotes for plain vanilla at-themoney options with the longest maturities available, i.e. typically 12 months.

Using the above-described methods we have been able to establish a reliable initial fair value estimate for precisely 300 of the PPNs in our database. ${ }^{15} 80$ PPNs - or about a fifth of the original sample - are thus lost at this point due to our inability to price these issues. Reasons for this may be that payoffs depend in a complicated way on interest rate dynamics (see again Skovmand and Jørgensen (2007) for an analysis of one particular issue), that the bond element is exposed to significant credit risk, that option terms are not sufficiently well described, or that necessary input parameters cannot be estimated.

Having thus priced both basic components of the structured bonds wherever possible, we obtain fair value estimates, $\widehat{P P N} N_{0}^{\text {Fair }}$, for a large part of the PPNs in our data set which can be compared with the corresponding issue prices, $P P N^{I s s u e}$.

Now, since the construction of PPNs clearly involves some costs, we expect to find a certain degree of overpricing of the bonds relative to their fair value. To quantify the degree of overpricing we therefore introduce the following measure of the estimated total cost percentage in relation to our PPNs,

$$
\widehat{T C}=\frac{P P N^{\text {Issue }}-\widehat{P P N_{0}^{\text {Fair }}}}{P P N^{\text {Issue }}} \cdot 100 \% .
$$

Note that we define and determine total costs as the estimated fair value deficit relative to the issue price of the structured bond. Alternatively one may use the fair value estimate, $\widehat{P P N} N_{0}^{\text {Fair }}$, in the denominator for a perhaps more standard measure of relative overpricing (as in e.g. Burth, Kraus, and Wohlwend (2001) and Stoimenov and Wilkens (2005)). However, since we want to compute and focus on a measure of the investors' costs, such a measure must be defined relative to the actual amount paid for the securities.

In practice and perhaps as a consequence of some of the aforementioned criticism directed towards PPNs, information regarding costs has increasingly often been disclosed with issues of PPNs. This is particularly the case for notes issued after January 1, 2008 when the

\footnotetext{
${ }^{15}$ As regards valuation of the option elements, historical volatilities (based on 180 daily observations) were used as input in 125 of these pricing problems, implied volatility estimates was used in 89 of the cases, and a combination of historical and implied volatilities were used in the remaining 86 cases.
} 
Danish Bankers Association (DBA) issued a recommendation (The Danish Bankers Association (2007)) to its members involved in the issuance of PPNs that all costs related to the issue should be disclosed to potential investors in the prospectus and in marketing material. It is explicitly stated in the DBA's recommendation that the disclosed costs should include fees to arranger and distributor, listing and marketing fees etc., and that the costs should be disclosed as an annual percentage rate of charge, i.e. as $\frac{\frac{T o t a l C o s t s}{P P N I s s u e}}{T} \cdot 100 \%$ where $T$ is the time to maturity of the PPN.

An annual percentage rate of charge, as defined above, is reported for 299 of the 380 PPNs in our sample and this statistic is of course included in our database, cf. also Table 3. Further, a total of 248 of these 299 PPNs are among the 300 structured bonds for which we have been able to estimate a fair theoretical value at issuance, cf. above. We are thus in possession of a subsample of 248 PPNs for which we have both an initial fair value estimate, an issue price, and a disclosed annual percentage rate of charge. Therefore it can be used to investigate, for example, whether estimated total costs deviate significantly from disclosed costs. To this end we define for PPNs a measure of unexplained or in effect hidden costs, $\widehat{H C}$, as

$$
\widehat{H C}=\widehat{T C}-D C
$$

where $\widehat{T C}$ is the total cost percentage defined in (7), and $D C$ is the disclosed cost percentage. The latter is calculated simply as the annual percentage rate of charge times the time to maturity of the PPN in accordance with the above-mentioned definition in The Danish Bankers Association (2007).

\section{Empirical results}

In the current section we first present and discuss some descriptive statistics regarding the two cost measures defined above. We then move on to test various hypotheses regarding the costs estimated for our sample of Danish PPNs, and we finally try to identify factors influencing these costs. The robustness of our results is also discussed. 


\subsection{An overview of estimated costs}

As explained in the previous section, we have been able to estimate total costs and hidden costs for a total of 300 and 248 PPNs, respectively. Figure 1 shows these cost estimates in simple histograms. As expected, a positive total cost is estimated for almost all products in our sample. ${ }^{16}$ More surprisingly we see that a large majority $(86 \%)$ of the hidden cost estimates are also positive. This is an unexpectedly large proportion given a natural base hypothesis that arrangers disclose all relevant costs and thus that hidden cost estimates should deviate from a $0 \%$ mean only due to random estimation errors. Hence Figure 1 calls for further investigation of the cost estimates, and in Tables 4 and 5 we have therefore provided more comprehensive and detailed descriptive statistics and some first diagnostic tests in relation to the sample of estimated costs.
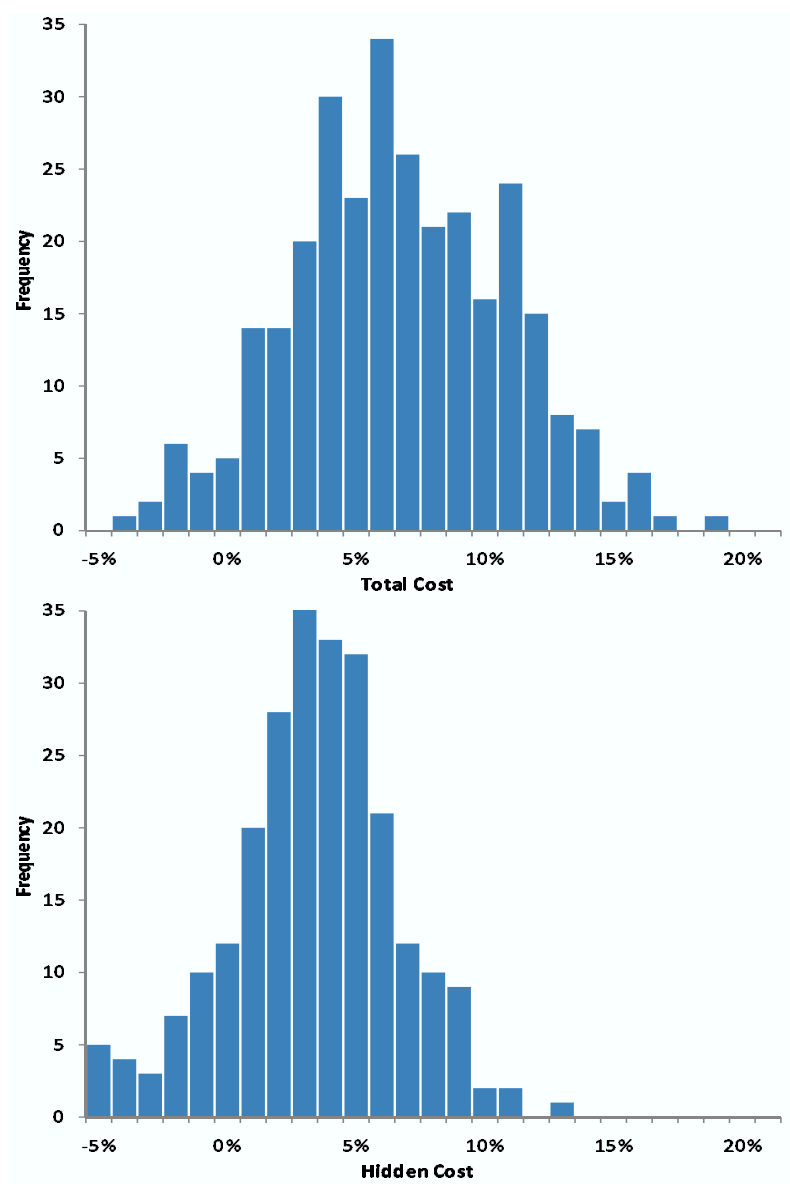

Figure 1: Histograms for total and hidden cost estimates

\footnotetext{
${ }^{16}$ To be more specific, a negative total cost is estimated for only 6 of the 300 PPNs.
} 
Table 4 relates to our total cost estimates, and Table 5 relates to our hidden cost sample. The first line of the tables reports descriptive statistics for the full samples. Next, and in order to get some first indications of what might explain the magnitude of the estimated costs, the tables categorize the samples according to ten different criteria and descriptive statistics are reported for different "values" of these criteria. The first categorization of the data is according to the issue period. In this category we divide the data set into products issued in two subperiods, the first of which spans from the beginning of 1998 and until the end of 2004, and the second being from the beginning of 2005 until the end of 2009. This split is not entirely arbitrary. Referring to Table, 2 it can be seen that the first subperiod may be characterized as a period where the market was in its infancy, whereas in the second period the PPN market had matured to a certain extent. To support this notion it can be noted that total nominal issues in the year 2005 alone were approximately equal to the sum of nominal issues in all previous years. It is thus natural to investigate whether this development and growth in the market have in some way had an effect on estimated costs.

\section{[Insert Tables 4 and 5 about here]}

The second and third categorizations in Tables 4 and 5 are according to whether the arranger and issuer are of "large", "medium", or "small" size." The motivation for this categorization could be a hypothesis that large arrangers/issuers would be able to negotiate better deals with distributors and/or investment banks (the option counterpart) and thus lower costs to investors. Similarly the nominal size of the individual PPN issues may lead to economics of scale so in the fourth categorization we sort PPNs into issue size quartiles. The fifth categorization concerns the (main) option type embedded in the particular product, and the sixth relates to the (main) type of underlying asset. In both of these cases one might reasonably conjecture that "simpler" could be less costly. The seventh, eighth, ninth, and tenth categorizations are done with respect to simple product characteristics such as whether the PPN is paying coupons or not, whether time to maturity is above 4 years or not (the average time to maturity is about 4 years, cf. Table 2), whether the option payoff is capped or not, and whether the option element contains a specific Asian element or not.

In addition to reporting descriptive statistics within each subcategory, we conduct Wilcoxon signed-rank tests for the null hypothesis of zero total/hidden costs in each subsample. Signif-

\footnotetext{
${ }^{17}$ Large, medium, and small size arrangers and issuers were defined and briefly discussed in Section 3.
} 
icance at the $1 \%$ level is indicated in the tables with ***, at the $5 \%$ level with $* *$, and with * at the $10 \%$ level. Finally, we conduct Kruskal-Wallis rank tests for differences in means between the subcategories. ${ }^{18}$ The $p$-values of these tests are reported in parentheses in the first line of each sub-panel. A significance here will suggest that this characteristic could in some form be tried as an explanatory variable in our later regressions, in which we will attempt to identify factors that can explain (parts of) the variation in estimated costs.

The most interesting conclusion from Table 4 for total costs is the following: We note first that both the mean and the median total costs based on the full sample are around $6 \%$. This is significantly different from zero (at the $1 \%$ level). Likewise it can be noted that means are significantly different from zero in all subcategories except for a few cases where the number of observations is so low (below 5 observations) that tests are basically meaningless due to lack of power.

The Kruskal-Wallis tests for differences in means of total costs indicate a number of things. First, it does indeed seem as if costs are lower in the more recent of the two subperiods. Second, larger arrangers/issuers are in general associated with lower costs than smaller and medium sized arrangers/issuers. A bit strangely though, medium sized arrangers seem to be associated with the highest total costs. Similarly the issue size quartile seems to be significantly negatively related to total costs (although this is only just significant at the $5 \%$ level), and total costs also seem to differ significantly between different option types with vanilla options (and simple spread options) apparently being less costly than all of the more exotic option types. Looking at total costs broken up with respect to the underlying asset(s) of the PPNs, it seems that currency and commodity based products are less costly than stock (index) based products. Whether the PPN has a coupon does not seem to matter, but longer products with a capped and/or an embedded Asian option element appear to cost more than short products without the same features.

Summing up the total cost analysis so far, we can say that PPNs from recent years, i.e. since the beginning of 2005, are in general associated with lower costs than PPNs from previous years. This is perhaps a consequence of increased volume and competition. Large single issues of PPNs also appear "cheaper" than smaller issues. Moreover, "simpler" products from larger

\footnotetext{
${ }^{18}$ Rigorously interpreted the Kruskal-Wallis test is a test for identical distributions. However, since the test is most sensitive to differences in location, it is frequently interpreted as a test of equal means against the alternative that at least one differs (Aczel (1999)). In cases with only two subcategories the conducted test for difference in means is the Wilcoxon two-sample test.
} 
arrangers/issuers are generally less costly than more complex products from smaller/medium sized market participants.

Turning now to Table 5 which analyzes the hidden cost estimates we emphasize the following conclusions: In the full sample consisting of 248 hidden cost estimates we observe an almost identical mean and median hidden cost of about $2.8 \%$. This is roughly half of total costs, but still highly significant. The change in perspective from total to hidden costs does not substantially affect the conclusions regarding the significance of costs when subcategories are considered. Costs are of course now reduced by the size of disclosed costs but remain highly significantly different from zero in most subcategories. Exceptions to this general picture again occur only when the number of observations in a subcategory is too low to conduct proper tests. As regards the difference-in-means tests within subcategories it can be observed from Table 5 that fewer product specific characteristics seem to affect hidden costs than total costs. For example, there is no significant difference in mean hidden costs in the two sub-periods considered. ${ }^{19}$ And although large arrangers/issuers still appear to impose lower costs than medium/small sized agents, this result is no longer statistically significant. The same is true when (quartiles of) nominal issue sizes are compared. The Asian option element factor also drops out, whereas the earlier "simplicity is cheaper" conclusion remains as regards the main option type, underlying asset, and the presence of a cap feature in the embedded option. PPNs with a time to maturity greater than 4 years are also still associated with higher costs than shorter products as far as hidden costs are concerned.

\subsection{Explaining costs}

Having thus presented a first analysis of our data and of some of its main characteristics, we now turn our attention towards developing a more complete model to explain what determines the costs associated with PPN investments in the Danish market. For this purpose we will in turn use our total and hidden cost estimates as the dependent variable in multiple regressions where we include essential issue specific characteristics of the PPNs as (potential) explanatory variables.

While some product characteristics - such as issue size and initial time to maturity -

\footnotetext{
${ }^{19} \mathrm{We}$ also tested for differences in mean costs in the subperiods before and after the issue of the DBA recommendation to disclose all costs in relation to PPN issues, i.e. before and after January 1, 2008 (see Section 4). The means of both total and hidden costs are lower after January 1, 2008, but not significantly so.
} 
are immediately usable as numerical variables in regressions, we must introduce a number of dummy variables to test the explanatory power of some of the more qualitative/"binary" characteristics. For instance, we introduce dummy variables for each of the issuer and arranger size categories, and we use dummies for the categories of option type and underlying asset type as well.

Some underlying indices for the embedded options are (baskets of) major stock indices such as S\&P500 and EuroStoxx50, while others are custom-made baskets of hand-picked individual stocks, for example from a particular industry. We also introduce dummies for these index types. Moreover, to test whether costs have changed since the inception of the Danish PPN market in early 1998, we use the time elapsed (in years) since January 1, 1998 as an independent variable in the regressions. A full list of variables that are tested for explanatory power in relation to our two cost measures is included in the Appendix.

Due to the large number of independent variables we proceed by implementing the method of stepwise regression. As described in many econometrics texts (such as e.g. Aczel (1999)), this is a systemized method of variable selection that starts out by identifying the most significant single-variable regression model. It then moves on to identify - from a list of proper candidate variables - the most significant additional variable according to a specified entry significance requirement, say $5 \%$. Next, the extended model is evaluated to determine whether the previously included variable(s) should be kept in the regression based again on a given significance requirement, and the procedure then moves on to check if further variables should be allowed to enter the regression model. This procedure of sequential testing for entry and exit of additional single variables continues until no further variables should be included in the regression and no variables inside the model can be dropped. ${ }^{20}$

With total costs as the dependent variable the stepwise (ordinary least squares) regression approach identifies the model for total costs (TC) given in Table 6 below.

\footnotetext{
${ }^{20} \mathrm{We}$ use $5 \%$ for both the "entry" and "exit" significance levels.
} 
Table 6: Regression model for Total Costs of Danish PPNs

\begin{tabular}{lcccccc}
\multicolumn{7}{c}{$\mathrm{TC}_{i}=\beta_{0}+\beta_{1} \mathrm{IYF}_{i}+\beta_{2} \mathrm{TTM}_{i}+\beta_{3} \mathrm{AM}_{i}+\beta_{4} \mathrm{CMI}_{i}+\beta_{5} \mathrm{CAP}_{i}+\epsilon_{i}$} \\
& $\beta_{0}$ & $\beta_{1}$ & $\beta_{2}$ & $\beta_{3}$ & $\beta_{4}$ & $\beta_{5}$ \\
\hline Estimate & 4.55 & -0.43 & 1.26 & 1.85 & 3.58 & 1.04 \\
Std.error & 0.75 & 0.08 & 0.10 & 0.36 & 0.45 & 0.37 \\
$p$-value & $<0.0001$ & $<0.0001$ & $<0.0001$ & $<0.0001$ & $<0.0001$ & 0.0055 \\
\hline $\mathrm{N}$ & 281 & & & & & \\
$R^{2}$ & 0.5446 & & & & &
\end{tabular}

Note: White heteroscedasticity consistent standard errors are used (no changes).

The White test for homoscedasticity is rejected with a $p$-value of 0.0383 .

As can be seen from Table 6, the stepwise regression procedure identifies five highly significant explanatory variables for total costs in addition to a highly significant intercept of 4.55\%. These are the issue date (IYF) measured in years from January 1, 1998, the initial time to maturity (TTM), the medium sized arranger dummy (AM), the custom-made index dummy (CMI), and finally a cap dummy (CAP).

The signs of the coefficients indicate that total costs have declined over time by approximately $0.4 \%$ per year during the period from 1998 to 2009 . The obvious interpretation of this is that the market has become more efficient over time. Second, it can be seen that costs increase with time to maturity by about $1.25 \%$ per year. The third regression coefficient suggests that PPNs issued by medium sized arrangers cost almost $2 \%$ more than products issued by other types of arrangers. ${ }^{21}$ Fourth, the sign on the CMI dummy indicates that products using custom-made indices are (much) more costly than more standard products. Finally, the cap dummy coefficient indicates that products equipped with rate of return caps bear an additional cost compared to products without a cap. The last two significant explanatory variables are clearly related to the complexity of the products. We will return to a more detailed discussion of these findings later in the paper.

We finally note from Table 6 that the $R^{2}$ of the regression is quite high (54\%), and that the number of observations has been reduced from 300 to 281 as a result of 19 outliers being

\footnotetext{
${ }^{21}$ This result is in large parts due to the influence from one particularly "expensive" arranger of medium size. We have tried assigning a dummy variable to this particular arranger. This dummy becomes highly significant with a coefficient of $1.44 \%$ in a regression where also the large arranger dummy enters with a highly significant negative coefficient. The remaining significant variables are as in Table 6 and with the same signs and of the

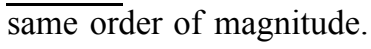


eliminated from the regression based on their Cook's distances being larger than $4 / N$ (see e.g. Chatterjee and Hadi (2006)). ${ }^{22}$

We next turn our attention to analyzing the sample of hidden costs and its possible explanatory variables among the product specifics. Running again the stepwise regression procedure we identify the following model and associated parameter estimates.

\section{Table 7: Regression model for Hidden Costs of Danish PPNs}

\begin{tabular}{lcccc} 
& \multicolumn{5}{c}{$\mathrm{HC}_{i}=\beta_{0}+\beta_{1} \mathrm{TTM}_{i}+\beta_{2} \mathrm{CMI}_{i}+\beta_{3} \mathrm{CAP}_{i}+\epsilon_{i}$} \\
& $\beta_{0}$ & $\beta_{1}$ & $\beta_{2}$ & $\beta_{3}$ \\
\hline Estimate & 0.04 & 0.65 & 2.25 & 0.95 \\
Std.error & 0.38 & 0.11 & 0.37 & 0.34 \\
$p$-value & 0.9239 & $<0.0001$ & $<0.0001$ & 0.0054 \\
\hline $\mathrm{N}$ & 234 & & \\
$R^{2}$ & 0.2772 \\
White heteroscedasticity consistent standard errors are used (no changes). \\
The White test for homoscedasticity is rejected with a $p$-value of 0.0224.
\end{tabular}

As can be seen from Table 7, we identify only three explanatory variables for hidden costs, namely initial time to maturity (TTM), the custom-made index dummy (CMI), and the cap dummy (CAP). All coefficients are positive and (except for the intercept) highly significant. The coefficients indicate that hidden costs increase by $0.65 \%$ per year in the PPNs original time to maturity. In addition a custom-made index implies a hidden cost of about $2.25 \%$. Finally, the coefficient on the CAP-variable suggests that the presence of a cap adds an additional percentage point of hidden costs. We might add that about a third of the sample (81 products) consists of relatively simple PPNs in the sense that both of the above-mentioned dummies have a value of 0 . Consequently it can be concluded that for a large part of the sample, hidden costs is simply a function of the products' time to maturity.23

We finally note that the regression's $R^{2}$ is $27 \%$ and that 14 outliers have been removed due to their Cook's distances, cf. earlier. Again, the removal of outliers has not affected the regression model in any significant way.

\footnotetext{
${ }^{22}$ The regression model identified using the full data sample has the same explanatory variables as the one presented in Table 6. Signs of coefficients are the same and of the same order of magnitude, but the $R^{2}$ is a bit lower $(40.7 \%)$.

${ }^{23}$ In a regression of the full sample of hidden cost estimates on the TTM-variable alone the $R^{2}$-coefficient drops to a mere $11 \%$, so CMI and CAP are indeed significant explanatory variables for the full sample.
} 


\subsection{Robustness of empirical results}

Above we have analyzed the percentage costs of PPNs in the Danish retail investment market and we have found a variety of highly significant results for what was defined as total costs and hidden costs, respectively. However, the costs analyzed were estimates determined in part via the adaptation of specific option pricing models along with necessary model parameter estimates. Therefore we must address the possibility that the cost estimates could be subject to systematic biases from either model error or errors in parameter estimation methods.

As regards the various versions of the Black-Scholes model that were implemented for pricing the option part of the PPNs, it is well-known that this framework and the assumptions underlying it are not perfect. The "volatility smile" for one is an empirical fact (see e.g. Rubinstein (1985) and Cont and Tankov (2004)) which contradicts the Black-Scholes assumptions and other imperfections have been documented as well. The Black-Scholes model nevertheless remains a market standard for quoting option prices (in terms of volatilities), and pricing biases from this model are usually modest for at-the-money options (see e.g. Hsieh and Ritchken (2005)) and almost by definition close to zero if market implied volatilities are used as inputs. It is therefore worth noting that all options in our sample are issued (and thus priced) as at-the-money options, and we also remind the reader that a large fraction of our sample was indeed priced using market implied volatility estimates either fully or in part (see footnote 15). While this makes us confident about the accuracy of the majority of our costs estimates, it remains to be checked if the cost estimates based mainly on historical volatility estimates constitute a source of bias in our results and if this is the cause of significance in our tests. The best way to conduct such a check is to perform a cost analysis in the subsample consisting only of those products for which the embedded option was priced using implied volatilities. This we will do below.

We first provide Table 8 which shows simple descriptive statistics for the two cost measures in relation to the subsample consisting only of the implied volatility (IV) priced PPNs. Total costs could in this case be estimated for a total of 86 products, and the hidden costs was estimated for 77 PPNs. 
Table 8: Cost percentages for PPNs priced via implied volatility estimates

\begin{tabular}{lrrrrrrr} 
& N & Mean & & Median & Std.Dev. & Min. & Max. \\
\hline \hline Total cost percentage & 86 & 4.00 & $* * *$ & 3.73 & 3.69 & -4.12 & 12.97 \\
\hline Hidden cost percentage & 77 & 1.44 & $* * *$ & 1.31 & 3.22 & -6.12 & 8.97
\end{tabular}

From Table 8 it is seen that mean total costs in the subsample amount to $4.00 \%$ (versus $6.17 \%$ in the full sample) and that the mean hidden costs are $1.44 \%$ (versus $2.83 \%$ in the full sample). These means are significantly different from the (higher) means in the rest of the sample. While it must thus be concluded that cost estimates obtained via option pricing models using implied volatility inputs are generally lower, we also see that both cost measures remain strongly significant. The result that there are significant total costs as well as hidden costs in PPNs is therefore quite robust, and it is not sensitive to the estimation methods used in relation to the parameter surrounded by the largest uncertainty, namely the volatility of the underlying index or indices.

It is worth emphasizing that while the degree of mispricing seems lower in the implied volatility priced subsample, there is no justification for concluding that we have underestimated option values in the products that were priced via historical volatility (or a combination of historical and implied volatility) estimates. Recall that we used implied volatility estimates whenever these were available. This was the case for the most standard and liquid underlying indices such as major stock indices and currency exchange rates. However, for more exotic underlyings where liquid option markets do not exist, we had to resort to historical volatility estimates. But the absence of liquid option markets is also likely to imply higher hedging costs for the arranger/issuer in relation to the option that has been shorted as part of the PPN package. So the difference in the level of estimated costs in our two samples may simply reflect the fact that these higher hedging costs are just passed on to investors.

For completeness we also provide Table 9 which contains the estimated regression model for total costs identified by the stepwise regression procedure using the implied volatility priced subsample: 
Table 9: Regression model for Total Costs in IV-priced subsample

\begin{tabular}{lccccc}
\multicolumn{5}{c}{$\mathrm{TC}_{i}=\beta_{0}+\beta_{1} \mathrm{AL}_{i}+\beta_{2} \mathrm{IL}_{i}+\beta_{3} \mathrm{TTM}_{i}+\beta_{4} \mathrm{CAP}_{i}+\epsilon_{i}$} \\
& $\beta_{0}$ & $\beta_{1}$ & $\beta_{2}$ & $\beta_{3}$ & $\beta_{4}$ \\
\hline Estimate & 0.56 & -2.11 & 1.98 & 1.29 & 3.51 \\
$p$-value & 0.6335 & 0.0027 & 0.0040 & 0.0004 & $<0.0001$ \\
\hline $\mathrm{N}$ & 81 & & & & \\
$R^{2}$ & 0.4761 & & & &
\end{tabular}

We note from Table 9 that the identification of explanatory variables for total costs (TC) is also quite robust to ways of estimating the volatility of the underlying index. We see again that arranger size matters - in this case highlighted by a negative coefficient on the large arranger dummy (AL). Also as earlier, the initial time to maturity (TTM) and the cap dummy (CAP) are highly significant with positive coefficients. That the large issuer dummy (IL) now enters the regression model with a positive coefficient is more of a puzzle. We see no logical explanation for this.

The regression model in Table 9 is based on 81 observations ( 5 outliers have been removed due to large Cook's distances) and the $R^{2}$ is about $48 \%$. We have also re-run the hidden cost regression on 72 observations. The results of this (not reported) are more mixed. The most significant coefficient in this regression is an intercept of $3.3 \%$.

\section{Conclusion}

We have presented in this paper a thorough analysis of the cost structure and pricing efficiency of a particularly large class of structured investment products from the Danish retail market known as Principal Protected Notes or PPNs. PPNs from the Danish retail market are remarkably similar in that they practically always comprise a simple (coupon or zero-coupon) bond and some kind of European-style option that will benefit from the appreciation of some underlying index. The two constituents of PPNs can be priced by theoretical models and the simple idea of this paper has been to investigate and compare the prices at which PPNs are initially offered and sold to investors with their initial theoretically true fair value. Our 
empirical study based on a data set consisting of almost 400 PPN issues in Denmark during the period from 1998 to 2009 has uncovered a variety of interesting results.

We first defined total costs as a measure of the PPNs' overpricing - or fair value deficit relative to the initial offer price, and we documented an average overpricing across all issues in the order of $6 \%$. That PPNs are overpriced relative to their fair value is not surprising since all the different agents participating in the design, construction, marketing, and sale of these products must be compensated for their efforts. The order of magnitude of the mispricing we find is in line with findings in studies of structured retail investment products in other markets. For example, Stoimenov and Wilkens (2005) find an average mispricing of 3.89\% in their study of the German market, and Szymanowska, Horst, and Veld (2009) find an average overpricing of $5.7 \%$ in their sample of reverse convertibles from the Dutch market.

The level of costs for PPN investments has been criticized in the past, and perhaps in an attempt to refute this criticism, arrangers/issuers have increasingly often disclosed costs of PPNs in their sales brochures and marketing material. Having also obtained data regarding these disclosed costs enabled us to define and calculate a measure of "net overpricing" of PPNs as the total costs less the disclosed costs. We called this hidden costs and found somewhat surprisingly - an average hidden cost of about 3\% for the 248 Danish PPNs that the data allowed us to price. If our theoretical pricing model is accurate, this means that on average only half of true total costs are disclosed to investors.

We next took the empirical analysis a step further and tried to link both total and hidden costs to a wide array of product specific characteristics via multivariate regression analysis. This analysis indicated that total costs have declined by about $0.4 \%$ per year during the data period 1998-2009, thus suggesting that the PPN market has become more efficient as it has developed and volume has increased. The regression analysis also established that time to maturity is a significant explanatory factor for the total costs of PPNs. We estimated that on average total costs increase by about $1.25 \%$ per year in a product's time to maturity. At first this is surprising since a priori there is little to justify that the actual costs of offering PPN investments should depend on the products' time to maturity. On the contrary, costs should be almost negligible after the time of issuance as no current portfolio management services are needed in connection with typical PPNs. However, an explanation for the result can perhaps be found in the fact that PPN arrangers/issuers often point to mutual funds when critics question the costs of PPNs. Mutual fund fees and costs normally amount to around 
$1-2 \%$ per year, which is of course nicely comparable with our estimated average total cost of $6 \%$ for PPNs with an average time to maturity of 4 years, i.e. approximately $1.5 \%$ per year. So in the absence of better explanations for PPN costs one might conjecture that PPN arrangers simply set costs and fees of PPNs at a level that can be justified by comparisons with a well-known retail investment alternative, namely mutual funds.

Total costs were also to some degree explained by arranger size with medium sized arrangers being significantly more costly - particularly in comparison with large arrangers. ${ }^{24}$ In addition we found evidence that PPNs with the embedded option based on customized indices are considerably more costly than products where the option's underlying index is more standard. This latter finding is likely explained by the fact that in practice it is indeed more costly for the arranger and issuer to provide - and hedge - options on specially designed, non-standard, and perhaps also illiquid indices than what is predicted by our idealized models.

The fifth and final explanatory variable identified for total costs was a dummy variable indicating whether the PPN option payoff was capped or not. The significant dummy coefficient indicated that PPNs with a capped option element bear higher costs and are thus more overpriced than non-capped products. Since a cap limits the upside potential for investors and thus depresses the option value, another way of stating this result is that in general PPN investors are not properly compensated - e.g. by a higher participation rate - when arrangers/issuers decide to cap a PPN. As suggested to us by a practitioner, one explanation for this finding may be that some PPNs are designed and priced first without taking the cap into account. The cap is then added sort of "in the last minute" as a "safety valve", i.e. to limit the issuer's risk from mis-estimated hedging costs. Such a practice - if it does in fact take place - should indeed lead to the identification of the cap dummy as a significant explanatory variable for total costs as in our regressions.

When we looked at hidden costs, our stepwise regression procedure identified a slightly simpler model where calendar time and arranger size dropped out of the final regression relation compared to the total cost regression model. So while total costs have declined over time, this does not seem to have been the case for hidden costs. In relation to the arranger size variables a tempting interpretation of the fact that the medium arranger dummy falls out of the hidden costs relation is that while medium sized arrangers have higher total costs, they

\footnotetext{
${ }^{24}$ Recall that we found a negative coefficient on the large arranger dummy in the IV-priced subsample, cf. Section 5.3.
} 
are at least being honest about it in their cost disclosures!

So the three remaining explanatory variables in the hidden cost regression are the time to maturity, the customized index dummy, and the cap dummy. In our view and interpretation this further strengthens the conjectures that time to maturity - and comparisons with mutual fund costs - is used as leverage for justifying higher fees, and that the addition of return caps is just another way of expropriating investor wealth at the time of issue.

The above-mentioned issues are among a large number of interesting questions in relation to the PPN market that could be investigated further in future research. Another obvious direction for future research would be to conduct a comprehensive study of the actual performance, i.e. mainly realized returns, of PPNs. Clearly it would be interesting to check if the overpricing documented in the present paper can - in full or in part - be seen as payment for arrangers'/issuers' skill in picking good investment cases (the "index") leading to superior performance of PPNs relative to other investments of similar risk category. On the other hand, if such a study finds evidence of poor risk-adjusted performance, then this would further support our conclusion that PPNs are overpriced at issuance. During the course of such an analysis future research could also contribute by developing theoretically solid risk measures and performance benchmarks for principal protected notes.

Finally, an interesting line for future research would be to study PPN pricing in the secondary market. As we have pointed out, this is no easy task since PPNs are not very liquid instruments. However, to the extent that reliable price quotes can be obtained, it would enable an important and relevant quality check of theoretical pricing models such as the ones implemented in this paper to estimate the fair value of PPNs. 


\section{References}

Aczel, A. D. (1999): Complete Business Statistics, McGraw-Hill, 4th edition.

Baule, R. and C. Tallau (2011): "The Pricing of Path-dependent Structured Financial Retail Products: The Case of Bonus Certificates," 21st Australasian Finance and Banking Conference 2008 Paper. Revised version available at www.ssrn.org.

Benet, B., A. Giannetti, and S. Pissaris (2006): "Gains from Structured Product Markets: The Case of Reverse-exchangeable Securities (RES)," Journal of Banking and Finance, 30(1):111-132.

Bennett, J. A., A. H. Chen, and P. McGuinness (1996): “An Analysis of Capital Guaranteed Funds," International Review of Economics and Finance, 5(3):259-268.

Bernard, C. and M. Ghossoub (2010): "Static Portfolio Choice under Cumulative Prospect Theory," Mathematics and Financial Economics, 2(4):277-306.

Bethel, J. and A. Ferrel (2006): "Policy Issues Raised by Structured Products," John M. Olin Center for Law, Economics, and Business, Discussion Paper No. 560, Harvard Law School.

Bjorrk, T. (2009): Arbitrage Theory in Continuous Time, Oxford University Press, 3rd edition.

Black, F. and M. Scholes (1973): "The Pricing of Options and Corporate Liabilities," Journal of Political Economy, 81(3):637-654.

Boyle, P. P. (1977): “Options: A Monte Carlo Approach,” Journal of Financial Economics, $4: 323-338$.

Branger, N. and B. Breuer (2008): “The Optimal Demand for Retail Derivatives," Working Paper, Finance Center Muenster, Muenster University.

Burth, S., T. Kraus, and H. Wohlwend (2001): "The Pricing of Structured Products in the Swiss Market," Journal of Derivatives, 9(2):30-40.

Chatterjee, S. and A. S. Hadi (2006): Regresssion Analysis by Example, John Wiley \& Sons, Hoboken, New Jersey. 
Chen, A. H. and J. W. Kensinger (1990): “An Analysis of Market-Index Certificates of Deposit," Journal of Financial Services Research, 4(2):93-110.

Chen, K. and R. S. Sears (1990): “Pricing the SPIN," Financial Management, 19(2):36-47.

Chen, K. and L. Wu (2007): “An Anatomy of Bullish Underlying Linked Securities,” Global Finance Journal, 18(1):34-46.

Cont, R. and P. Tankov (2004): Financial Modeling with Jump Processes, Chapman \& Hall/CRC, Boca Raton, Florida.

Das, S. (2006): Structured Products Vol. 1 and Vol. 2, Wiley, Singapore, 3rd edition.

FASB (2004): Project Update: Fair Value Measurements, March 5, 2004, Financial Accounting Standards Board. Available at www. fasb.org.

Grunbichler, A. and H. Wohlwend (2005): "The Valuation of Structured Products: Empirical Findings for the Swiss Market," Financial Markets and Portfolio Management, 19(4):361380.

Henderson, B. and N. Pearson (2007): "Patterns in the Payoffs of Structured Equity Derivatives," AFA 2008 New Orleans Meetings Paper.

Hens, T. and M. O. Rieger (2008): “The Dark Side of the Moon: Structured Products from the Customer's Perspective," Working Paper, Swiss Finance Institute, University of Zurich. Hsieh, K. C. and P. Ritchken (2005): “An Empirical Comparison of GARCH Option Pricing Models," Review of Derivatives Research, 8(3):129-150.

Hull, J. C. (2009): Options, Futures, and other Derivatives, Prentice-Hall, Inc., 7th edition. IASB (2004): "International Financial Reporting Standard 4," Available at www. iasb.org.

Kat, H. M. (2001): Structured Equity Derivatives, Wiley, London, United Kingdom.

Margrabe, W. (1978): "The Value of an Option to Exchange One Asset for Another," Journal of Finance, XXXIII(1):177-186. 
Rasmussen, A.-S. R. (2007): “Indekserede Obligationer," Kvartalsoversigt. Danmarks Nationalbank, (2):47-61. In Danish.

Rathgeber, A. and Y. Wang (2010): “Market Pricing of Credit Linked Notes,” EFMA 2010 Annual Meeting Paper.

Rubinstein, M. (1985): “Nonparametric Tests of Alternative Option Pricing Models," Journal of Finance, 40:455-480.

Ruf, T. (2010): "The Bank Always Wins: The Dynamics of Overpricing in Structured Products," 2011 MFA Annual Meeting Paper.

Scott, W. R. (2003): Financial Accounting Theory, Prentice Hall, Pearson Education Canada Inc., Toronto, Ontario, 3rd edition.

Skovmand, D. and P. L. Jørgensen (2007): "The Valuation of Callable Bonds with Floored CMS-spread Coupons,” WILMOTT, pages 106-125.

Stoimenov, P. A. and S. Wilkens (2005): “Are Structured Products 'Fairly' Priced? An Analysis of the German Market for Equity-Linked Instruments," Journal of Banking and Finance, 29(12):2971-2993.

Szymanowska, M., J. T. Horst, and C. Veld (2009): "Reverse Convertible Bonds Analyzed," Journal of Futures Markets, 29(10):895-919.

The Danish Bankers Association (2007): Finansradets anbefalinger om informationer $i$ forbindelse med salg og markedsføring af strukturerede obligationer (The Danish Bankers Association's recommendations on information in connection with the sale and marketing of structured bonds), The Danish Bankers Association. December 3rd, 2007. In Danish.

Tversky, A. and D. Kahneman (1992): “Advances in Prospect Theory: Cumulative Representation of Uncertainty," Journal of Risk and Uncertainty, 5(4):297-323.

Wallmeier, M. and M. Diethelm (2009): "Market Pricing of Exotic Structured Products: The Case of Multi-Asset Barrier Reverse Convertibles in Switzerland," Journal of Derivatives, 17(2):59-72. 
Wasserfallen, W. and C. Schenk (1996): "Portfolio Insurance for the Small Investor in Switzerland," Journal of Derivatives, 3(3):37-43.

Wilkens, S., C. Erner, and K. Roder (2003): "The Pricing of Structured Products in Germany," Journal of Derivatives, 11(1):55-69. 


\section{Appendix: List of variables}

\begin{tabular}{|c|c|c|}
\hline Name & Description & Scale \\
\hline \multicolumn{3}{|c|}{ Dependent variables } \\
\hline $\mathrm{TC}$ & Total cost (see equation (7)) & Percent \\
\hline $\mathrm{HC}$ & Hidden cost (see equation (8)) & Percent \\
\hline \multicolumn{3}{|c|}{ Explanatory variables } \\
\hline IYF & Yearfrac from 1998-01-01 to the issue date & Number of years (two decimals) \\
\hline $\mathrm{AL}$ & Large Arranger (2 largest) & $1=$ Large, $0=$ Not large \\
\hline $\mathrm{AM}$ & Medium arranger (3rd-6th largest) & $1=$ Medium, $0=$ Not medium \\
\hline AS & Small arranger (rest) & $1=$ Small, $0=$ Not small \\
\hline IL & Large issuer (2 largest) & $1=$ Large, $0=$ Not large \\
\hline IM & Medium issuer (3rd-5th largest) & $1=$ Medium, $0=$ Not medium \\
\hline IS & Small issuer (rest) & $1=$ Small, $0=$ Not small \\
\hline O_BASKET & Basket option & $1=$ Basket, $0=$ Not basket \\
\hline O_ASIAN & Asian option & $1=$ Asian, $0=$ Not Asian \\
\hline O_BARRIER & Barrier option & $1=$ Barrier, $0=$ Not barrier \\
\hline O_VANILLA & Vanilla option & $1=$ Vanilla, $0=$ Not vanilla \\
\hline O_SPREAD & Spread option & $1=$ Spread, $0=$ Not spread \\
\hline O_LOOKBACK & Lookback option & $1=$ Lookback, $0=$ Not Lookback \\
\hline O_HIMALAYA & Himalaya option & 1=Himalayan, $0=$ Not Himalayan \\
\hline STOCKINDEX & Major stock index/indices & $1=$ Stock index, $0=$ Not stock index \\
\hline CURRENCY & Currencies & $1=$ Currency, $0=$ Not Currency \\
\hline CMI & Basket of hand-picked single stocks & $1=$ Custom index, $0=$ Not custom index \\
\hline COMBINATION & Combination of several asset types & $1=$ Combination, $0=$ Not combination \\
\hline COMMODITY & Commodities & $1=$ Commodity, $0=$ Not commodity \\
\hline FUND & Fund or mutual fund & $1=$ Fund, $0=$ Not fund \\
\hline REALESTATE & Real estate & $1=$ Real Estate, $0=$ Not Real estate \\
\hline COUPON & Coupon or zero-coupon bond & $1=$ Coupon, $0=$ Zero-coupon \\
\hline TTM & Lifespan of the product & Number of years (two decimals) \\
\hline ASIAN & Does the product have an Asian element & $1=$ Asian element, $0=$ No Asian element \\
\hline CAP & Does the product have a cap & $1=$ Cap, $0=$ No cap \\
\hline $\mathrm{OP}$ & Issued before or after 2008-01-01 & $1=$ After, $0=$ Before \\
\hline NOM_DKK & Nom. issue in DKK & Amount i mDKK \\
\hline
\end{tabular}


Table 4: Descriptive statistics and simple tests for the total cost estimates. $p$-values in parentheses are for Kruskal-Wallis rank tests for difference in means within the group. Stars indicate significance of costs based on a Wilcoxon signed-rank test at the $1 \%(* * *), 5 \%(* *)$, and $10 \%$ level $(*)$.

\begin{tabular}{|c|c|c|c|c|c|c|c|}
\hline & $\mathrm{N}$ & Mean & & Median & Std.Dev. & Min. & Max. \\
\hline All & 300 & 6.17 & $* * *$ & 5.86 & 4.16 & -4.12 & 18.80 \\
\hline \multicolumn{8}{|c|}{ Issue period $(\mathrm{p}<0.0001)$} \\
\hline $1998-2004$ & 76 & 8.19 & $* * *$ & 8.13 & 4.23 & -2.97 & 18.80 \\
\hline $2005-2009$ & 224 & 5.49 & $* * *$ & 5.42 & 3.92 & -4.12 & 15.13 \\
\hline \multicolumn{8}{|c|}{ Arranger $(\mathrm{p}<0.0001)$} \\
\hline Large & 143 & 4.76 & $* * *$ & 4.81 & 3.44 & -4.12 & 12.26 \\
\hline Medium & 84 & 8.23 & $* * *$ & 8.48 & 4.00 & -2.97 & 16.96 \\
\hline Small & 73 & 6.57 & $* * *$ & 6.17 & 4.60 & -3.08 & 18.80 \\
\hline \multicolumn{8}{|l|}{ Issuer $(p=0.0305)$} \\
\hline Large & 62 & 5.08 & $* * *$ & 4.55 & 3.56 & -2.29 & 12.97 \\
\hline Medium & 42 & 6.45 & $* * *$ & 6.18 & 4.86 & -3.08 & 18.80 \\
\hline Small & 196 & 6.46 & $* * *$ & 6.47 & 4.14 & -4.12 & 16.96 \\
\hline \multicolumn{8}{|c|}{ Nom. issue size $(p=0.0472)$} \\
\hline Quartile 1 & 75 & 6.42 & $* * *$ & 6.36 & 4.30 & -2.27 & 18.80 \\
\hline Quartile 2 & 75 & 6.76 & $* * *$ & 6.86 & 4.71 & -4.12 & 16.96 \\
\hline Quartile 3 & 75 & 6.35 & $* * *$ & 5.72 & 4.15 & -1.60 & 15.69 \\
\hline Quartile 4 & 75 & 5.17 & $* * *$ & 5.32 & 3.27 & -2.95 & 12.26 \\
\hline \multicolumn{8}{|c|}{ Option type $(p=0.0003)$} \\
\hline Basket & 143 & 6.86 & $* * *$ & 6.90 & 3.91 & -3.08 & 15.13 \\
\hline Asian & 65 & 6.37 & $* * *$ & 5.78 & 4.24 & -2.95 & 18.80 \\
\hline Barrier & 29 & 5.63 & $* * *$ & 4.78 & 4.29 & 1.23 & 16.96 \\
\hline Vanilla & 42 & 3.93 & $* * *$ & 3.34 & 3.44 & -3.29 & 12.26 \\
\hline Spread & 8 & 4.25 & $* *$ & 4.27 & 4.29 & -2.63 & 12.10 \\
\hline Lookback & 11 & 7.24 & $* * *$ & 7.10 & 6.13 & -4.12 & 15.69 \\
\hline Himalaya & 2 & 7.99 & & 7.99 & 3.95 & 5.19 & 10.78 \\
\hline \multicolumn{8}{|c|}{ Underlying asset $(\mathrm{p}<0.0001)$} \\
\hline Stock index & 98 & 7.10 & $* * *$ & 6.98 & 3.90 & -3.08 & 18.80 \\
\hline Currency & 111 & 4.17 & $* * *$ & 3.63 & 3.57 & -4.12 & 13.39 \\
\hline Customized index & 55 & 8.99 & $* * *$ & 8.59 & 3.73 & -1.89 & 16.96 \\
\hline Combination & 14 & 7.21 & $* * *$ & 8.03 & 3.58 & 1.05 & 13.11 \\
\hline Commodity & 14 & 4.44 & $* * *$ & 4.89 & 3.48 & -2.97 & 12.26 \\
\hline Fund & 3 & 1.44 & & -2.29 & 6.87 & -2.76 & 9.36 \\
\hline Real estate & 4 & 4.90 & & 4.83 & 0.83 & 4.07 & 5.84 \\
\hline Mutual fund & 1 & 12.26 & & & & & \\
\hline \multicolumn{8}{|l|}{ Coupon $(\mathrm{p}=0.0779)$} \\
\hline No coupon & 252 & 6.30 & $* * *$ & 6.21 & 4.12 & -4.12 & 18.80 \\
\hline Coupon & 48 & 5.50 & $* * *$ & 4.22 & 4.36 & -0.09 & 15.69 \\
\hline \multicolumn{8}{|c|}{ Time to maturity $(\mathrm{p}<0.0001)$} \\
\hline $0-4$ years & 230 & 5.28 & $* * *$ & 5.25 & 3.94 & -4.12 & 16.96 \\
\hline$>4$ years & 70 & 9.11 & $* * *$ & 9.40 & 3.46 & -1.89 & 18.80 \\
\hline \multicolumn{8}{|l|}{ Cap $(\mathrm{p}<0.0001)$} \\
\hline No cap & 212 & 5.61 & $* * *$ & 5.28 & 4.32 & -4.12 & 18.80 \\
\hline Cap & 88 & 7.53 & $* * *$ & 7.36 & 3.40 & -1.52 & 16.96 \\
\hline \multicolumn{8}{|l|}{ Asian $(\mathrm{p}<0.0001)$} \\
\hline No Asian element & 125 & 4.94 & $* * *$ & 3.90 & 4.18 & -4.12 & 16.96 \\
\hline Asian element & 175 & 7.05 & $* * *$ & 7.10 & 3.93 & -3.08 & 18.80 \\
\hline
\end{tabular}


Table 5: Descriptive statistics and simple tests for the hidden cost estimates. $p$-values in parentheses are for Kruskal-Wallis rank tests for difference in means within the group. Stars indicate significance of costs based on a Wilcoxon signed-rank test at the $1 \%(* *), 5 \%(* *)$, and $10 \%$ level $(*)$.

\begin{tabular}{|c|c|c|c|c|c|c|c|}
\hline & $\mathrm{N}$ & Mean & & Median & Std.Dev. & Min. & Max. \\
\hline All & 248 & 2.83 & $* * *$ & 2.84 & 3.28 & -8.29 & 12.59 \\
\hline \multicolumn{8}{|c|}{ Issue period $(\mathrm{p}=0.3828)$} \\
\hline $1998-2004$ & 51 & 3.29 & $* * *$ & 3.07 & 3.53 & -5.19 & 12.59 \\
\hline $2005-2009$ & 197 & 2.71 & $* * *$ & 2.81 & 3.21 & -8.29 & 9.27 \\
\hline \multicolumn{8}{|c|}{ Arranger $(p=0.0799)$} \\
\hline Large & 128 & 2.48 & $* * *$ & 2.46 & 2.89 & -6.12 & 9.27 \\
\hline Medium & 70 & 3.10 & $* * *$ & 3.46 & 3.60 & -8.29 & 10.96 \\
\hline Small & 50 & 3.35 & $* * *$ & 3.68 & 3.68 & -5.45 & 12.59 \\
\hline \multicolumn{8}{|l|}{ Issuer $(p=0.0867)$} \\
\hline Large & 60 & 2.19 & $* * *$ & 2.19 & 2.86 & -8.29 & 8.97 \\
\hline Medium & 31 & 2.90 & $* * *$ & 3.07 & 4.20 & -5.45 & 12.59 \\
\hline Small & 157 & 3.06 & $* * *$ & 3.24 & 3.21 & -6.12 & 10.96 \\
\hline \multicolumn{8}{|c|}{ Nom. issue size $(p=0.3728)$} \\
\hline Quartile 1 & 62 & 2.89 & $* * *$ & 3.34 & 3.35 & -5.19 & 8.97 \\
\hline Quartile 2 & 62 & 3.10 & $* * *$ & 3.64 & 3.92 & -8.29 & 10.96 \\
\hline Quartile 3 & 62 & 2.91 & $* * *$ & 2.54 & 3.34 & -4.55 & 12.59 \\
\hline Quartile 4 & 62 & 2.42 & $* * *$ & 2.62 & 2.34 & -3.61 & 7.76 \\
\hline \multicolumn{8}{|c|}{ Option type $(\mathrm{p}=0.0031)$} \\
\hline Basket & 117 & 3.40 & $* * *$ & 3.92 & 3.02 & -5.45 & 9.27 \\
\hline Asian & 51 & 1.81 & $* * *$ & 2.00 & 3.30 & -8.29 & 8.39 \\
\hline Barrier & 24 & 3.89 & $* * *$ & 3.37 & 3.27 & -1.79 & 10.96 \\
\hline Vanilla & 40 & 1.82 & $* * *$ & 1.88 & 2.81 & -6.07 & 7.76 \\
\hline Spread & 6 & 1.43 & & 2.16 & 2.61 & -3.61 & 3.32 \\
\hline Lookback & 10 & 3.64 & $*$ & 3.07 & 5.61 & -6.12 & 12.59 \\
\hline \multicolumn{8}{|l|}{ Himalaya } \\
\hline \multicolumn{8}{|c|}{ Underlying asset $(\mathrm{p}<0.0001)$} \\
\hline Stock index & 70 & 2.97 & $* * *$ & 3.27 & 2.74 & -5.45 & 8.65 \\
\hline Currency & 97 & 1.64 & $* * *$ & 1.54 & 2.95 & -6.12 & 8.97 \\
\hline Customized index & 54 & 5.11 & $* * *$ & 5.09 & 3.44 & -5.19 & 12.59 \\
\hline Combination & 9 & 3.43 & $* * *$ & 3.42 & 1.59 & 0.79 & 6.11 \\
\hline Commodity & 11 & 1.44 & $*$ & 1.44 & 2.06 & -1.79 & 5.16 \\
\hline Fund & 2 & -1.09 & & -1.09 & 10.19 & -8.29 & 6.11 \\
\hline Real estate & 4 & 1.66 & & 1.69 & 0.55 & 1.07 & 2.24 \\
\hline Mutual fund & 1 & 7.76 & & & & & \\
\hline \multicolumn{8}{|l|}{ Coupon $(p=0.8750)$} \\
\hline No coupon & 210 & 2.78 & $* * *$ & 3.03 & 3.26 & -8.29 & 10.96 \\
\hline Coupon & 38 & 3.12 & $* * *$ & 2.46 & 3.40 & -3.26 & 12.59 \\
\hline \multicolumn{8}{|c|}{ Time to maturity $(\mathrm{p}=0.0076)$} \\
\hline $0-4$ years & 194 & 2.56 & $* * *$ & 2.63 & 3.29 & -8.29 & 10.96 \\
\hline$>4$ years & 54 & 3.82 & $* * *$ & 4.27 & 3.06 & -5.19 & 12.59 \\
\hline \multicolumn{8}{|l|}{ Cap $(\mathrm{p}<0.0001)$} \\
\hline No cap & 170 & 2.12 & $* * *$ & 2.18 & 3.28 & -8.29 & 12.59 \\
\hline Cap & 78 & 4.37 & $* * *$ & 4.55 & 2.70 & -4.02 & 10.96 \\
\hline \multicolumn{8}{|l|}{ Asian $(p=0.1668)$} \\
\hline No Asian element & 107 & 2.67 & $* * *$ & 2.50 & 3.54 & -6.12 & 12.59 \\
\hline Asian element & 141 & 2.95 & $* * *$ & 3.10 & 3.07 & -8.29 & 9.13 \\
\hline
\end{tabular}

